\title{
Advantage of PET/CT in Target Delineation of MRI- negative Cervical Lymph Nodes In Intensity-Modulated Radiation Therapy Planning for Nasopharyngeal Carcinoma
}

Guanzhu Shen ${ }^{1 *}$, Weiwei Xiao ${ }^{2 *}$, Fei Han², Wei Fan ${ }^{3}$, Xiao-ping Lin ${ }^{3}$, Lixia Lu², Lie Zheng4, Ning Yue ${ }^{5}$, Bruce Haffty ${ }^{5}$, Chong Zhao ${ }^{\varpi}$, Xiaowu Deng ${ }^{2 \varpi}$

1. Department of Radiation Oncology, Affiliated Cancer Hospital \& Institute of Guangzhou Medical University,78 Hengzhigang Road, Guangzhou 510095, China;

2. Department of Radiation Oncology, Sun Yat-sen University Cancer Center, State Key Laboratory of Oncology in South China, Collaborative Innovation Center of Cancer Medicine, Guangzhou, 510060, P.R. China;

3. Department of Nuclear Medicine, Sun Yat-sen University Cancer Center, State Key Laboratory of Oncology in South China, Collaborative Innovation Center of Cancer Medicine, Guangzhou, 510060, P.R. China;

4. Department of Imaging, Sun Yat-sen University Cancer Center, State Key Laboratory of Oncology in South China, Collaborative Innovation Center of Cancer Medicine, Guangzhou, 510060, P.R. China.

5. Rutgers Cancer Institute of New Jersey, 195 Little Albany Street, New Brunswick, NJ, USA.

${ }^{*}$ Guanzhu Shen and Weiwei Xiao contributed equally to this work.

$\triangle$ Corresponding authors: Chong Zhao, Department of Radiation Oncology, Sun Yat-sen University Cancer Center, State Key Laboratory of Oncology in South China, Collaborative Innovation Center of Cancer Medicine, 651 Dong Feng Road East, Guangzhou 510060, China Tel: +86-20-87342638 Fax: +86-20-87342638 Email: zhaochong@sysucc.org.cn And Xiaowu Deng, Department of Radiation Oncology, Sun Yat-sen University Cancer Center, State Key Laboratory of Oncology in South China, Collaborative Innovation Center of Cancer Medicine, 651 Dong Feng Road East, Guangzhou 510060, China Tel: +86-20-87343044 Fax: +86-20-87343044 Email: dengxw@mail.sysu.edu.cn

(c) Ivyspring International Publisher. This is an open access article distributed under the terms of the Creative Commons Attribution (CC BY-NC) license (https://creativecommons.org/licenses/by-nc/4.0/). See http://ivyspring.com/terms for full terms and conditions.

Received: 2017.06.22; Accepted: 2017.10.13; Published: 2017.11.06

\begin{abstract}
Introduction: In intensity-modulated radiation therapy (IMRT) planning for nasopharyngeal carcinoma (NPC), cervical lymph nodes (CLNs) that appear negative on magnetic resonance imaging (MRI) scans can be difficult to target. The purpose of this study was to assess the advantage of 18F-fluorodeoxyglucose positron emission tomography with computed tomography (18F-FDG PET/CT) for distinguishing MRI-negative CLNs and the effect of 18 F-FDG PET/CT on diagnosis, target delineation, and dose prescription in IMRT planning for NPC.

Methods: Thirty-five NPC patients with 37 MRI-negative CLNs underwent 18F-FDG PET/CT imaging before treatment. Ultrasonography-guided fine-needle aspiration cytology (USgFNAC) was performed to examine the pathology of CLNs. The 18F-FDG PET/CT and cytopathological results were compared, and the diagnostic accuracy of 18 F-FDG PET/CT was calculated. The cytopathologically confirmed CLNs were delineated and treated as the gross tumor volume of lymph nodes (denoted as GTVnd).

Results: Nineteen of the $37 \mathrm{MRI}$-negative CLNs were positive on $18 \mathrm{~F}$-FDG PET/CT, and metastasis was confirmed by USgFNAC in 16 CLNs. Of the remaining 18 18F-FDG PET/CT-negative lymph nodes, metastasis was confirmed in one. The diagnostic accuracy, sensitivity, and specificity of $18 \mathrm{~F}-\mathrm{FDG}$ PET/CT were $89.2 \%, 94.1 \%$, and $85.0 \%$, respectively. The positive and negative predictive values were $84.2 \%$ and $94.4 \%$, respectively. With a median follow-up of 48.3 months, no relapse was observed among the 18F-FDG PET/CT-positive CLNs with metastasis confirmed by USgFNAC and treated as GTVnd.

Conclusion: 18F-FDG PET/CT had high accuracy, sensitivity, and specificity for distinguishing MRI-negative CLNs. 18F-FDG PET/CT-positive CLNs could reasonably be categorized as high-risk clinical tumor volume in IMRT planning for NPC.
\end{abstract}

Key words: ${ }^{18} \mathrm{~F}-\mathrm{FDG}$ PET/CT; MRI; nasopharyngeal carcinoma; IMRT, cervical lymph nodes. 


\section{Introduction}

The nasopharyngeal region has many lymphatic vessels, increasing the likelihood of nasopharyngeal carcinoma (NPC) spreading via vessels. The cervical lymph node (CLN) metastasis rate is $\sim 70-85 \%$ in newly diagnosed patients with NPC [1, 2]. Radiation therapy is the primary treatment choice for NPC. Because of the proximities of many critical organs in the head and neck, intensity-modulated radiation therapy (IMRT) is the preferred modality based on its superiority in providing conformal dose coverage for target volumes while sparing adjacent normal structures. Because of the highly conformal dose distribution and sharp dose drop of IMRT, correct identification and accurate delineation of the target volumes, including the primary nasopharynx gross tumor volume (GTVnx) and the positive CLNs (GTVnd), is extremely important. Because of its advantages for soft tissue differentiation and resolution, magnetic resonance imaging (MRI) is widely used to improve the accuracy of target identification and delineation in NPC. Nevertheless, various studies have shown that small metastases can exist in MRI-negative lymph nodes. Such metastases could be missed during cervical target delineation, leading to insufficient dose coverage and treatment failures in NPC and other head and neck cancers [3-7].

${ }^{18}$ F-fluorodeoxyglucose positron emission tomography-computed tomography $\left({ }^{18} \mathrm{~F}-\mathrm{FDG}\right.$ PET/CT) combines anatomical and functional imaging and has the potential to improve the sensitivity of detecting small CLN metastases in patients with NPC. Small MRI-negative metastases in CLNs can appear as positive on ${ }^{18}$ F-FDG PET/CT due to abnormal glucose uptake $[4,8,9]$. To the best of our knowledge, for target volume delineation of IMRT planning, there is no consensus on how to define CLNs that are MRI-negative.

The purpose of this study was to assess the advantage of ${ }^{18} \mathrm{~F}-\mathrm{FDG}$ PET/CT in distinguishing MRI-negative CLNs in NPC by comparing the results of ${ }^{18} \mathrm{~F}-\mathrm{FDG}$ PET/CT imaging and cytopathological examinations. In addition, we evaluated how this advantage might affect the target volume delineation of IMRT planning for NPC.

\section{Methods}

This study was undertaken at the Sun Yat-sen University Cancer Center. Non-disseminated patients with NPC underwent IMRT with or without chemotherapy as per the standard guidelines. The patients were evaluated using MRI before enrollment. Solitary and negative CLNs (with the shortest diameter of the maximum cross-section $\geq 5 \mathrm{~mm}$ ) found at different levels from metastatic CLNs were identified, and ${ }^{18}$ F-FDG PET-CT was performed. Exclusion criteria were as follows: (1) other combined or primary malignancies at the time of diagnosis; (2) previous history of malignancy; (3) previous treatment with any anti-tumor therapy; (4) history of neck surgery; (5) history of diabetes or other metabolic diseases; (6) platelet count < $100 \times 10^{9} / \mathrm{L}$; and (7) abnormal coagulation function. The pathology of MRI-negative CLNs was examined by pretreatment ultrasonography-guided fine-needle aspiration cytology (USgFNAC). The study was approved by the ethics committee of Sun Yat-sen University Cancer Center, and the participants provided written informed consent before treatment.

MRI was performed with a 1.5 T superconducting magnetic resonance imaging system (Signa Excite II 1.5; GE Medical Systems, Waukesha, WI, USA) using conventional spin-echo (SE) sequences. The scan parameters were as follows: T1WI TR $=400-600 \mathrm{~ms}$, TE $=15-25 \mathrm{~ms}$; T2WI TR = 800-3000 ms, TE $=90-150 \mathrm{~ms}$; flip angle $90^{\circ}$, slice thickness/interslice interval of $5 \mathrm{~mm} / 1 \mathrm{~mm}$; matrix $256 \times 256$, and FOV $210 \mathrm{~mm}$. A standard orthogonal head coil was used. The axial scan sets covered the region from the suprasellar cistern to the sternoclavicular joint. An intravenous bolus injection of gadolinium-diethylenetriamine pentaacetic acid (gadolinium-EDTA, Gd-DTPA; $0.1 \mathrm{mmol} / \mathrm{kg}$ ) was administered, and T1W1 imaging was acquired followed plain scans. The MRI images were then independently reviewed by two experienced radiologists (LLX and ZL).

The diagnostic criteria for positive CLNs were based on those of van den Brekel et al. [10] and included swollen lymph nodes with the shortest diameter $\geq 10 \mathrm{~mm}$ ( $\geq 11 \mathrm{~mm}$ for level IIa) in the largest axis or with capsular invasion, or $>3$ lymph nodes with a cluster- or string-like distribution, or the presence of central necrosis. Other CLNs were defined as MRI-negative. If the diagnosis was inconsistent between the two radiologists, a consensus was reached after discussion.

${ }^{18} \mathrm{~F}-\mathrm{FDG}$ PET/CT imaging was performed using a combined PET/CT scanner (Discovery ST-16, GE Health Care, Piscataway, NJ, USA). The ${ }^{18}$ F-FDG radiochemical purity was $>95 \%$. Before scanning, patients fasted for at least 6 hours; scanning was performed when the patients had a blood glucose level of $\leq 8.1 \mathrm{mmol} / \mathrm{L}$. The ${ }^{18} \mathrm{~F}-\mathrm{FDG}$ dose was 5.55 $\mathrm{MBq} / \mathrm{kg}(0.15 \mathrm{mCi} / \mathrm{kg})$. After intravenous injection of ${ }^{18} \mathrm{~F}-\mathrm{FDG}$, the patients rested quietly in bed in a darkened room for 45-60 min and then underwent PET/CT imaging after a bowel movement and urination. The range of acquisition was from the 
calvarium to the middle of the femur. The CT scanning parameters were as follows: tube voltage 140 $\mathrm{kV}$, automatic modulation of the tube current across a range of 30-150 mA, a gantry rotation speed of $0.8 \mathrm{~s}$, and a pitch of $1.375: 1$. The PET scanning parameters were as follows: two-dimensional (2D) mode, 5-7 bed positions, $2.5 \mathrm{~min} /$ bed position, matrix $128 \times 128$, and slice thickness $3.25 \mathrm{~mm}$. The three-dimensional (3D) reconstruction was performed using the ordered subset expectation maximization iterative algorithm. The acquired images were transferred to a GE Xeleris workstation and were independently reviewed by two experienced nuclear medicine physicians (FW and LXP). A diagnosis of CLN metastasis was based on the distribution of ${ }^{18} \mathrm{~F}-\mathrm{FDG}$ in the CLNs and surrounding normal tissue, lesion morphology, and the maximum standardized uptake value (SUVmax) of the region of interest. Lymph nodes were considered PET/CT-positive based on location, intensity, shape, size, and visual correlation with CT images to differentiate physiologic uptake from pathologic uptake. Positivity was also considered if: (1) abnormal increased FDG uptake that was greater than the surrounding background of blood pool activity, and (2) the SUVmax of the lymph node was > 2.5 [11-13]. If the initial diagnosis differed between the two physicians, discussions were held to reach a consensus.

USgFNAC biopsy was performed after MRI and ${ }^{18} \mathrm{~F}-\mathrm{FDG}$ PET/CT imaging. The locations of the CLNs were determined using a conventional ultrasonography system (Nemio17, Toshiba Medical Systems Corporation, Otawara, Tochigi, Japan). To ensure CLN presence and accurate ultrasonography guidance, patients were selected for biopsy based on the previously acquired MRI and PET/CT images as those having a solitary CLN $\geq 5 \mathrm{~mm}$ on MRI, without a cluster- or string-like distribution. After routine disinfection and draping, local anesthesia was performed using 1\% lidocaine. An automatic ejection biopsy gun (Bard MAGNVM, Biopsy Instrument, Covington, GA, USA) and 16-G or 18 G-needles (lengths $16 \mathrm{~cm}$ and $20 \mathrm{~cm}$, respectively) were used to perform the biopsies. Biopsies were performed under ultrasonography guidance, and a tissue specimen with a length of 15 or $22 \mathrm{~mm}$ was obtained from each insertion. For each patient, up to six specimens were collected, and all collected tissue strips were placed on sterilized filter paper. The tissue strips were fixed in $10 \%$ formaldehyde solution and sent for routine cytopathological examination.

Details of radiotherapy planning have been described previously [14]. Simultaneous modulated accelerated-boost radiation therapy was implemented for all patients, with prescription doses of $68-70$
Gy/30 fractions (F) to the planning target volume (PTV) of the GTVnx (PGTVnx), 60-66 Gy/30 F to the PTV of the GTVnd (PGTVnd), 60 Gy/30 F to the PTV of the clinical tumor volume (CTV1, PTV1), and 54 Gy/30 F to the PTV of the low-risk CTV, termed CTV2 (PTV2). All CLNs that were confirmed positive by USgFNAC were delineated as the GTVnd and were prescribed 60-66 Gy/30 fractions.

\section{Data analysis and follow-up}

Based on the ${ }^{18} \mathrm{~F}-\mathrm{FDG}$ PET/CT examination and cytopathological results, MRI-negative CLNs were divided into four categories as follows: true positive (TP), both PET/CT and cytopathological examination showed metastasis; true negative (TN), both PET/CT and cytopathological examination were negative for malignancy; false positive (FP), PET/CT was positive and cytopathological examination was negative; false negative (FN), PET/CT was negative and cytopathological examination was positive. The accuracy, sensitivity, specificity, positive predictive value (PPV), and negative predictive value (NPV) of ${ }^{18}$ F-FDG PET/CT were calculated.

Patients were followed-up monthly for the first 3 months, every 3 months for the next 3 years, every 6 months for the next 2 years, and then annually. Lymph node relapse was diagnosed by MRI or ${ }^{18}$ F-FDG PET/CT and confirmed by USgFNAC.

\section{Results}

Patients were enrolled from June 2005 to August 2012. Thirty-five newly diagnosed NPC patients with 37 MRI-negative CLNs underwent whole-body 18F-FDG PET/CT scanning, and USgFNAC biopsy was applied for all MRI-negative CLNs. There were 24 males and 11 females, and the median age was 46 years (range, 28-65 years). The patients' clinical features are presented in Table 1.

All 37 MRI-negative CLNs were solitary (not having a cluster- or string-like distribution). The majority (70\%) of CLNs were located at Level II. The mean shortest diameter of the maximum cross-section was $7.1 \mathrm{~mm}$ (5-9 mm). ${ }^{18} \mathrm{~F}-\mathrm{FDG}$ PET/CT imaging identified 19 positive findings among the 37 MRI-negative CLNs, with a mean SUVmax of 6.8 (range, 2.6-14.9), details of these CLNs are shown in Table 2 . The remaining 18 lymph nodes were negative on ${ }^{18} \mathrm{~F}-\mathrm{FDG}$ PET/CT, with a mean SUVmax of 2.0 (range, 0-6.1). Figure 1 shows an example of an MRI-negative lymph node that was positive on ${ }^{18}$ F-FDG PET/CT imaging. The cytopathological results confirmed metastasis in 16 of the $19{ }^{18} \mathrm{~F}-\mathrm{FDG}$ PET/CT-positive CLNs, showing an accuracy of $84.2 \%$ for distinguishing malignant lymph nodes from MRI-negative CLNs. The cytopathological diagnosis 
for the remaining 3 nodes was lymphadenitis. Among the 18 negative ${ }^{18} \mathrm{~F}-\mathrm{FDG}$ PET/CT findings, cytopathological examination confirmed inflammatory changes or normal lymph node tissue in 17 lymph nodes and metastasis in 1 . The diagnostic accuracy, sensitivity, and specificity of $18 \mathrm{~F}-\mathrm{FDG}$ PET/CT were $89.2 \%, 94.1 \%$, and $85 \%$, respectively. The PPV and NPV were $84.2 \%$ and $94.4 \%$, respectively.

Table 1. Clinical features of 35 newly diagnosed patients with NPC

\begin{tabular}{lll}
\hline & Classification & Number of patients \\
\hline Sex & Male & 24 \\
& Female & 11 \\
Age range (years) & $28-65$ (median: & 46 years) \\
Histologic classification (WHO) & I + IIa & 3 \\
& IIb & 32 \\
T classification* & T1 & 3 \\
& T2 & 9 \\
& T3 & 18 \\
N classification* & T4 & 5 \\
& N0 & 16 \\
& N1 & 9 \\
Clinical stage* $^{*}$ & N2 & 10 \\
& N3 & 0 \\
& I & 3 \\
II & 8 \\
III & 19 \\
IV & 5 \\
\hline
\end{tabular}

*Staging according to the 2002 American Joint Committee on Cancer criteria.

Table 2. Details of the 19 PET/CT-positive CLNs

\begin{tabular}{lllll}
\hline No. & Levels & Diameter $(\mathrm{mm})$ & SUVmax & USgFNAC Results* $^{*}$ \\
\hline 1 & I & 6.0 & 4.3 & $\mathrm{P}$ \\
2 & I & 8.0 & 5 & $\mathrm{P}$ \\
3 & I & 8.0 & 3.5 & $\mathrm{P}$ \\
4 & II & 7.0 & 3 & $\mathrm{P}$ \\
5 & II & 6.0 & 6.4 & $\mathrm{P}$ \\
6 & II & 8.0 & 4.4 & $\mathrm{P}$ \\
7 & II & 9.0 & 12.8 & $\mathrm{P}$ \\
8 & II & 7.0 & 12.8 & $\mathrm{P}$ \\
9 & II & 8.0 & 11.7 & $\mathrm{P}$ \\
10 & II & 7.0 & 4.3 & $\mathrm{~N}$ \\
11 & II & 7.0 & 3.2 & $\mathrm{P}$ \\
12 & II & 9.0 & 5.6 & $\mathrm{P}$ \\
13 & II & 5.0 & 13.2 & $\mathrm{P}$ \\
14 & II & 9.0 & 6.2 & $\mathrm{~N}$ \\
15 & II & 9.0 & 2.6 & $\mathrm{P}$ \\
16 & III & 5.0 & 3.3 & $\mathrm{P}$ \\
17 & III & 9.0 & 8.3 & $\mathrm{~N}$ \\
18 & V & 5.0 & 3.9 & $\mathrm{P}$ \\
19 & V & 6.0 & 14.9 & $\mathrm{P}$ \\
\hline
\end{tabular}

CLN, cervical lymph node; SUVmax, maximum standardized uptake value USgFNAC, ultrasonography-guided fine-needle aspiration cytology; $\mathrm{P}$, Positive; $\mathrm{N}$, Negative

Detailed dose distribution analyses showed that the average mean dose to these CLNs was $68.02 \mathrm{~Gy}$ (63.45-74.50 Gy). Follow-up was terminated on December 31, 2015. With a median follow-up of 48.3 months (27.3-120.9 months), 3 patients exhibited lymph node recurrence with 2 recurrences at Level III and 1 at the parotid lymph node. The MRI-negative CLNs of these 3 patients were all located at Level II; therefore, these recurrences were not at the level of the previously treated CLNs.

\section{Discussion}

Regional relapse is one of the main failure patterns for NPC patients treated with IMRT $[15,16]$. The correct diagnosis, accurate delineation of metastatic CLNs, and radical radiation of such nodes are extremely important for the success of radiotherapy, especially when using IMRT. Because of the high imaging resolution of soft tissue, MRI is becoming the preferred reference imaging modality for the delineation of target volumes in patients with NPC [17-19]. However, the morphological diagnostic criteria for lymph nodes used for MRI are based on a single cutoff value with presumed high sensitivity and specificity. For CLNs, diagnostic criteria for malignant lymph nodes have been proposed by van den Brekel et al. [10], with the shortest diameter of swollen lymph nodes $\geq 10 \mathrm{~mm}$ ( $\geq 11 \mathrm{~mm}$ in level IIa) being used most commonly. However, previous studies showed that metastases could exist in MRI-negative CLNs, and $>40 \%$ of CLN metastases occur in lymph nodes with a diameter $<10 \mathrm{~mm}$ [20-22]. In the present study, the prevalence of "occult" lymph node metastase in CLNs $<10 \mathrm{~mm}$ was $45.9 \%$ (17/37). Therefore, diagnosis based on the size of the CLN could result in misdiagnosis of metastatic lymph nodes.

Previous studies in patients with NPC and other head and neck cancers have indicated that ${ }^{18} \mathrm{~F}-\mathrm{FDG}$ PET/CT was superior to CT and MRI for metastasis detection in CLNs [23-25]. However, the superiority of ${ }^{18} \mathrm{~F}-\mathrm{FDG}$ PET/CT for MRI-negative lymph nodes has not been demonstrated clearly. The present study evaluated the usefulness of ${ }^{18} \mathrm{~F}-\mathrm{FDG}$ PET/CT for MRI-negative CLNs in patients with NPC. The diagnostic sensitivity and specificity of ${ }^{18} \mathrm{~F}-\mathrm{FDG}$ PET/CT were $94.1 \%$, and $85 \%$, respectively. PET has limitations in detecting small tumor volumes, which are associated with the spatial resolution of current PET cameras and partial volume effects. Crippa et al. [26] reported that the average reconstructed axial resolution of FDG PET was $5.3 \mathrm{~mm}$. The FN case in the present study could be a result of such limitations, but might also be due to a relatively low number of cancer cells in the CLN. Only lymph nodes with the shortest diameter of the maximum cross-section of $\geq 5$ $\mathrm{mm}$ on MRI were evaluated, which might have contributed to the sensitivity and specificity values in the present study. USgFNAC was used for the 
pathological examinations. A comparison of USgFNAC and neck dissection for head and neck cancer patients showed that USgFNAC was a highly accurate technique for the investigation of CLN metastases [27].

Previous studies have reported relatively low PPVs; it was $61.6 \%$ in Lee et al. [28] and 50\% in Schöder et al. [21]. In the present study, there were 3 FPs on ${ }^{18} \mathrm{~F}-\mathrm{FDG}$ PET/CT, and the PPV was $84.2 \%$ (16/19). All 3 FPs had inflammatory hyperplastic tissue. The relatively high PPV and small sample size in the present study might explain the non-conformity of the diagnostic criteria with that reported by previous studies. The small number of FPs might also limit the reliability of the comparison between TPs and FPs; therefore, these data will need to be validated in a larger patient population.

Without resorting to additional diagnostic means, determination of an appropriate treatment strategy for MRI-negative CLNs could be difficult because of the uncertainty of the metastatic involvement status of CLNs. Li et al. [29] suggested that any node could be defined as a suspicious metastatic node if it does not meet the diagnostic criteria on MRI but has a shortest axial diameter of at least $8 \mathrm{~mm}$. In addition, MRI-negative grouped nodes could be defined as suspicious metastatic nodes if at least 1 of the nodes has a minimal dimension of $5 \mathrm{~mm}$. However, we found that metastasis in CLNs that were solitary on MRI and had a shortest axial diameter of < $8 \mathrm{~mm}$. A recent study by Peng et al. found that a total biological effective dose of 72 Gy to MRI-negative CLNs provided a clinical benefit [30]. The present study revealed that $18 \mathrm{~F}-\mathrm{FDG}$ PET/CT was highly accurate for detecting metastasis in MRI-negative CLNs and could provide valuable information for the delineation of target volumes in IMRT treatment of NPC.

The target volume definitions in IMRT mainly follow the recommendations of the related International Commission on Radiation Units and
Measurements (ICRU) reports for the area of radiotherapy. In the latest ICRU report \#83 [31], a subclinical malignant disease with a certain probability $(5-10 \%)$ of occult disease should be considered relevant for therapy and are defined in the CTV. Subclinical malignant disease includes possible regional infiltration into lymph nodes, despite their normal appearance on clinical and radiological examinations. In this study, the accuracy of ${ }^{18} \mathrm{~F}-\mathrm{FDG}$ PET/CT for distinguishing malignant lymph nodes from MRI-negative CLNs was $84.2 \%$. Despite the relatively small sample size, the probability of $84.2 \%$ is much higher than that of $5-10 \%$ suggested by the ICRU report \#83. In the IMRT treatment planning for NPC, the current practice is to divide the CTV into CTV1, which includes high-risk sites of microscopic tumor extension, and CTV2, which covers low-risk sites of microscopic tumor extension and the lymphatic drainage area of positive CLNs. Different radiation doses are prescribed to each of these CTVs. We believe that it is reasonable to categorize positive CLNs as high-risk CTVs in the IMRT plans to ensure they are treated with a higher prescribed dose. In the present study, all the ${ }^{18} \mathrm{~F}-\mathrm{FDG}$ PET/CT-positive CLNs that were confirmed as metastatic by USgFNAC were delineated as the GTVnd and treated with a high dose, and none of these CLNs relapsed. In current clinical practice, the target volume GTVnd is typically referred to as MRI-positive CLNs that meet the positive diagnosis criteria of MRI/CT. Therefore, questions remain as to whether the category of MRI-negative but ${ }^{18} \mathrm{~F}-\mathrm{FDG}$ PET/CT-positive CLNs with high odds of metastasis should be defined as GTVnd in IMRT treatment of NPC. We believe that USgFNAC alone, to confirm or rule out CLN metastasis, would not be sufficiently rigorous compared with lymph node resection. However, the present study had a small sample size. Therefore, the true clinical benefit of adding PET/CT in nodal staging will need to be evaluated in a large prospective study.
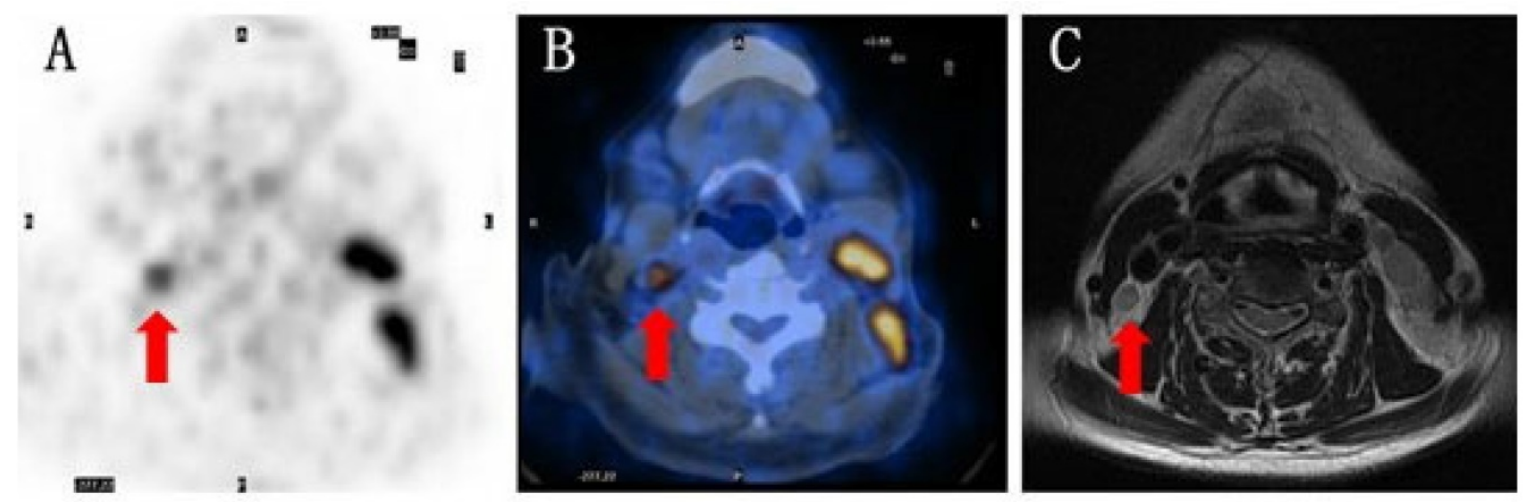

Figure 1. A representative example of imaging findings A) 59-year-old female with condensed uptake at the site of the lymph node suggested by pre-treatment PET. B) Suspected malignant lymph nodes revealed by 18F-FDG PET/CT scan. C) Negative MRI diagnosis. 


\section{Conclusion}

${ }^{18}$ F-FDG PET/CT had high accuracy, sensitivity, and specificity for distinguishing MRI-negative CLNs in patients with NPC. Almost half of the MRI-negative CLNs in the present study were positively involved and presented metastasis risk. It is reasonable to categorize these MRI-negative but ${ }^{18}$ F-FDG PET/CT-positive CLNs as CTV1 in IMRT planning for NPC.

\section{Abbreviations}

IMRT: intensity-modulated radiation therapy; NPC: nasopharyngeal carcinoma; CLNs: cervical lymph nodes; MRI: magnetic resonance imaging; ${ }^{18} \mathrm{~F}-\mathrm{FDG}$ PET/CT: ${ }^{18} \mathrm{~F}$-fluorodeoxyglucose positron emission tomography; USgFNAC: ultrasonographyguided fine-needle aspiration cytology; SUVmax: maximum standardized uptake value; ICRU: International Commission on Radiation Units and Measurements; GTVnx: primary nasopharynx gross tumor volume; GTVnd: positive neck lymph nodes; CTV: clinical tumor volume; CTV1: high-risk clinical tumor volume; CTV2: low-risk clinical tumor volume; PTV: planning target volume; PGTVnx: planning target volume of primary nasopharynx gross tumor volume; PGTVnd: planning target volume of positive neck lymph nodes; PTV1: planning target volume of high-risk clinical tumor volume; PTV2: planning target volume of low-risk clinical tumor volume; TP: true positive; TN: true negative; FP: false positive; FN: false negative; PPV: positive predictive value; NPV: negative predictive value.

\section{Acknowledgments}

No companies participated in the protocol development, running of the trial, or analysis of the results.

\section{Funding}

This work was jointly supported by the National Key R\&D Program of China (2017YFC0113200), Science and Technology program of Guangdong Province, China (2015B020214002), Science and Technology program of Guangzhou, China (201508020105), and Guangzhou key medical discipline construction project, China.

\section{Competing Interests}

The authors have declared that no competing interest exists.

\section{References}

1. Wang $\mathrm{X}, \mathrm{Hu} \mathrm{C}$, Ying $\mathrm{H}$, et al. Patterns of lymph node metastasis from nasopharyngeal carcinoma based on the 2013 updated consensus guidelines for neck node levels. Radiother Oncol. 2015; 115: 41-5.
2. Mao YP, Liang SB, Liu LZ, et al. The $\mathrm{N}$ staging system in nasopharyngeal carcinoma with radiation therapy oncology group guidelines for lymph node levels based on magnetic resonance imaging. Clin Cancer Res. 2008; 14: 7497-503.

3. Don DM, Anzai Y, Lufkin RB, et al. Evaluation of cervical lymph node metastases in squamous cell carcinoma of the head and neck. Laryngoscope. 1995; 105: 669-74.

4. Kao CH, Hsieh JF, Tsai SC, et al. Comparison of 18-fluoro-2-deoxyglucose positron emission tomography and computed tomography in detection of cervical lymph node metastases of nasopharyngeal carcinoma. Ann Otol Rhinol Laryngol. 2000;109: 1130-4.

5. van den Brekel MW, van der Waal I, Meijer CJ, et al. The incidence of micrometastases in neck dissection specimens obtained from elective neck dissections. Laryngoscope. 1996; 106: 987-91.

6. Sigg MB, Steinert H, Gratz K, et al. Staging of head and neck tumors: [18F]fluorodeoxyglucose positron emission tomography compared with physical examination and conventional imaging modalities. J Oral Maxillofac Surg. 2003; 61: 1022-9.

7. Braams JW, Pruim J, Freling NJ, et al. Detection of lymph node metastases of squamous-cell cancer of the head and neck with FDG-PET and MRI. J Nucl Med 1995; 36: 211-6.

8. Yen TC, Chang YC, Chan SC, et al. Are dual-phase 18F-FDG PET scans necessary in nasopharyngeal carcinoma to assess the primary tumour and loco-regional nodes? Eur J Nucl Med Mol Imaging. 2005; 32: 541-8.

9. Chang JT, Chan SC, Yen TC, et al. Nasopharyngeal carcinoma staging by (18)F-fluorodeoxyglucose positron emission tomography. Int J Radiat Oncol Biol Phys. 2005; 62:501-7.

10. van den Brekel MW, Stel HV, Castelijns JA, et al. Cervical lymph node metastasis: assessment of radiologic criteria. Radiology. 1990; 177: 379-84.

11. Kunawudhi A, Pak-art R, Keelawat S, et al. Detection of subcentimeter metastatic cervical lymph node by 18F-FDG PET/CT in patients with well-differentiated thyroid carcinoma and high serum thyroglobulin but negative 131I whole-body scan. Clin Nucl Med. 2012; 37: 561-7.

12. Ozer E, Naiboglu B, Meacham R, et al. The value of PET/CT to assess clinically negative necks. Eur Arch Otorhinolaryngol. 2012; 269: 2411-4.

13. Yoon DY, Hwang HS, Chang SK, et al. CT, MR, US,18F-FDG PET/CT, and their combined use for the assessment of cervical lymph node metastases in squamous cell carcinoma of the head and neck. Eur Radiol. 2009; 19: 634-42.

14. Sun $\mathrm{X}, \mathrm{Su} \mathrm{S}, \mathrm{Chen} \mathrm{C}$, et al. Long-term outcomes of intensity-modulated radiotherapy for 868 patients with nasopharyngeal carcinoma: an analysis of survival and treatment toxicities. Radiother Oncol 2014; 110: 398-403.

15. Ng WT, Lee MC, Hung WM, et al. Clinical outcomes and patterns of failure after intensity-modulated radiotherapy for nasopharyngeal carcinoma. Int J Radiat Oncol Biol Phys. 2011; 79: 420-8.

16. Xue $\mathrm{F}, \mathrm{Hu} \mathrm{C}, \mathrm{He} \mathrm{X}$. Long-term patterns of regional failure for nasopharyngeal carcinoma following intensity-modulated radiation therapy. J Cancer. 2017; 8: 993-9.

17. $\mathrm{Ng} \mathrm{SH}$, Chang TC, Ko SF, et al. Nasopharyngeal carcinoma: MRI and CT assessment. Neuroradiology 1997; 39: 741-6.

18. Olmi P, Fallai C, Colagrande $S$, et al. Staging and follow-up of nasopharyngeal carcinoma: magnetic resonance imaging versus computerized tomography. Int J Radiat Oncol Biol Phys. 1995; 32: 795-800.

19. Sakata K, Hareyama M, Tamakawa M, et al. Prognostic factors of nasopharynx tumors investigated by MR imaging and the value of MR imaging in the newly published TNM staging. Int J Radiat Oncol Biol Phys. 1999; 43: 273-8.

20. Cetin B, Atasever T, Akdemir UO, et al. The role of positron emission tomography with 18F-fluorodeoxyglucose in nodal staging of clinical and radiological N(0) head and neck cancers. Eur Arch Otorhinolaryngol. 2013; 270: 2307-13.

21. Schoder H, Carlson DL, Kraus DH, et al. 18F-FDG PET/CT for detecting nodal metastases in patients with oral cancer staged N0 by clinical examination and CT/MRI. J Nucl Med. 2006; 47: 755-62.

22. Eichhorn $\mathrm{T}$, Schroeder HG, Glanz $\mathrm{H}$, et al. [Histologically controlled comparison of palpation and sonography in the diagnosis of cervical lymph node metastases]. Laryngol Rhinol Otol (Stuttg). 1987; 66: 266-74.

23. Suenaga Y, Kitajima K, Kanda T, et al. 18F-FDG PET/CT imaging for detection of nodal metastases in patients with squamous cell carcinoma of the pharynx and larynx: comparison with CT. Jpn J Radiol. 2016; 34: 203-10.

24. Lai $\mathrm{V}$, Khong PL. Updates on MR imaging and 18F-FDG PET/CT imaging in nasopharyngeal carcinoma. Oral Oncol. 2014; 50: 539-48.

25. Hempel JM, Kloeckner R, Krick S, et al. Impact of combined FDG-PET/CT and MRI on the detection of local recurrence and nodal metastases in thyroid cancer. Cancer Imaging. 2016; 16: 37

26. Crippa F, Leutner M, Belli F, et al. Which kinds of lymph node metastases can FDG PET detect? A clinical study in melanoma. J Nucl Med. 2000; 41:1491-4.

27. Knappe M, Louw M, Gregor RT. Ultrasonography-guided fine-needle aspiration for the assessment of cervical metastases. Arch Otolaryngol Head Neck Surg. 2000; 126: 1091-6.

28. Lee $\mathrm{SH}$, Huh $\mathrm{SH}$, Jin $\mathrm{SM}$, et al. Diagnostic value of only 18F-fluorodeocyglucose positron emission tomography/computed tomography-positive lymph nodes in head and neck squamous cell carcinoma. Otolaryngol Head Neck Surg. 2012; 147: 692-8.

29. Li JG, Yuan X, Zhang LL, et al. A randomized clinical trial comparing prophylactic upper versus whole-neck irradiation in the treatment of patients 
with node-negative nasopharyngeal carcinoma. Cancer-Am Cancer Soc. 2013; 119: 3170-6.

30. Peng $\mathrm{H}$, Chen $\mathrm{L}$, Guo $\mathrm{R}$, et al. Clinical treatment considerations in the intensity-modulated radiotherapy era for patients with N0-category nasopharyngeal carcinoma and enlarged neck lymph nodes. Chin J Cancer. 2017; 36: 32.

31. International Commission on Radiation Units and Measurements. Prescribing, recording, and reporting photon-beam intensity-modulated radiation therapy (IMRT): contents. J ICRU. 2010; 10 